\section{Wird die Ehe besser - geht es auch den Männerherzen besser}

Das kardiale Risikoprofil verheirateter Männer verbessert sich, wenn die Beziehung zur Ehefrau besser wird. 620 Teilnehmer der Avon Longitudinal Study of Parents and Children wurden zu Studienbeginn, nach sechs und nach 18 Jahren untersucht und zu ihrer Ehe befragt. Die Qualität der Beziehung (gleichbleibend gut oder gleichbleibend schlecht) hatte keinen Einfluss auf das kardiale Risikoprofil. Hatte sich das Verhältnis zur Gattin hingegen verbessert, war das LDL-Cholesterin nach 18 Jahren um 0,25 mmol/I niedriger als bei Männern in konstant guter Ehe. Auch der BMI $(-1,07 \mathrm{~kg} / \mathrm{m} 2)$, das Gesamtcholesterin $(-0,24 \mathrm{mmol} / \mathrm{l})$ und der diastolische Blutdruck $(-2,24$ $\mathrm{mmHg}$ ) waren tendenziell verbessert. Hatte sich die Ehe verschlechtert, schlug sich dies in einem erhöhten diastolischen Blutdruck nieder $(+2,74 \mathrm{mmHg})$ nieder.

J Epidemiol Community Health 2017

\section{Gestationsdiabetes: Folgen nach der Schwangerschaft?}

Ob ein Gestationsdiabetes später kardiovaskuläre Ereignisse nach sich zieht, lässt sich durch den Lebensstil nach der Schwangerschaft beeinflussen. Fast 90.000 Teilnehmerinnen der Nurses' Heath Study II, die mindestens einmal schwanger waren, wurden regelmäßig zu Lebensstil und Gesundheit befragt. Bei 1,55\% der 5.292 Frauen mit Schwangerschaftsdiabetes kam es im Lauf von 25 Jahren zu einem Herzinfarkt oder Schlaganfall, bei den übrigen betrug die Rate $1,28 \%$. Damit war das relative Risiko für ein solches Ereignis bei Frauen mit Gestationsdiabetes um $43 \%$ höher als in der Vergleichsgruppe. Wurde allerdings bezüglich des Lebensstils nach der Schwangerschaft adjustiert, war das Risiko nur noch um 29\% erhöht. Durch eine gesunde Lebensweise haben Frauen mit Gestationsdiabetes gute Chancen, ihre kardiovaskuläre Prognose zu normalisieren, so die Autoren.

JAMA Intern Med 2017 wusstseinstrübungen. Maximal möglich sind sechs Punkte, ab fünf Punkten beträgt die Dreimonatsmortalität etwa $40 \%$.

Angesichts der gravierenden Folgen von Deliren bei alten Menschen stehe die Prävention im Vordergrund. Hüll verwies auf das in den USA entwickelte multimodale Interventionsprogramm HELP (Hospital Elder Life Program). Dieses ist mittlerweile auch in einigen deutschen Kliniken implementiert worden. In Studien ließ sich damit die Delirprävalenz zum Teil halbieren. Es zielt unter anderem darauf, den Stress für Delir-gefährdete Patienten in Kliniken zu reduzieren: So sollten Katheter so früh wie möglich entfernt, akustische und optische Störungen vermieden werden. Hüll nannte etwa piepsende und blin- kende Geräte oder eine Dauerbeleuchtung. Hilfreich seien dagegen Brillen und Hörgeräte, damit sich die Betroffenen besser orientieren können. Eine regelmäßige Überprüfung der Flüssigkeitsund Nahrungszufuhr, ein zurückhaltender Gebrauch von psychotropen Medikamenten, eine frühe Mobilisierung und ausreichende kognitive Stimulation sowie ein regelmäßiges Screening auf Delirsymptome sind entscheidende $\mathrm{Be}$ standteile des Programms. Gefördert werden sollten zudem nichtpharmakologische Ansätze zur Schlafförderung und Angstvermeidung.

(TM)

Symposium „Gerontopsychiatrie: Depression und Delir". Weltkongress der World Psychiatric Association in Kooperation mit der DGPPN; Berlin, 8.-12.10.2017.

\title{
Bald als App: Trainingsprogramm für junge Alte
} Eine Smartphone-App soll jungen Alten ab 60 Jahren helfen, mehr körperli-
che Aktivität in ihren Alltag zu integrieren. Das Konzept wird derzeit in einer
Studie geprüft.

W er ältere Menschen zu mehr körperliche Aktivität bewegen will, darf nicht erwarten, dass sie von einem Tag auf den anderen mit Joggen, Radfahren oder Wandern beginnen. Gefragt sind daher niederschwellige Angebote, die sich gut in den Alltag integrieren lassen. Vor einigen Jahren haben Forscher ein entsprechendes Programm für ältere Menschen entwickelt. Mit dem „Lifestyle Integrated Functional Exercise Program“ (LiFE) ließ sich das Sturzrisiko um etwa ein Drittel senken.

Die Übungen wurden für Menschen über 70 Jahre konzipiert. Besser wäre es jedoch, ältere Menschen würden schon mit dem Training beginnen, bevor sie hinfallen oder ihren Alltag nicht mehr gut bewältigen können. Forscher der Universität Heidelberg versuchen das Programm nun an jüngere Ältere ab 60 Jahre anzupassen. Sie haben in einem europäischen Verbundprojekt eine Version für diese Altersgruppe entwickelt, die sie auch als Smartphone- und Smartwatch-App verfügbar machen wollen (eLiFE). Ältere Menschen üben damit unter anderem, auf Zehenspitzen zu gehen, beim Treppensteigen zwei Stufen auf einmal zu nehmen, auf Spaziergängen Objekte zu überspringen oder auf einem Bein stehend den Oberkörper und das andere Bein waagerecht zu halten. Diese Übungen gibt es in unterschiedlichen Schwierigkeitsstufen - die Teilnehmer können sich mit der Zeit also steigern, erläuterte Dr. Michael Schwenk, Netzwerk Altersforschung in Heidelberg. Über die elektronische Version lassen sich nicht nur Videos abrufen, welche die einzelnen Übungen erläutern, die Teilnehmer können ihre Fortschritte mit den Geräten dokumentieren. Die App erinnert an das Training oder erfasst per Bewegungssensoren die körperliche Aktivität. Sollte sich das eLiFE-Konzept bewähren, könnte sich in naher Zukunft vielleicht jeder Interessent die entsprechende App auf sein Smartgerät laden und mit dem Training loslegen.

(TM)

Symposium „Bewegung und körperliches Training in den Alltag integrieren: Neue Interventionsstrategien". 29. Jahreskongress der DGG; Frankfurt/M., 28.-30.9.2017. 